\title{
Voltammetric characterization of grafted polymer electrode self modification with carbon nanotubes (GPESMCNT)
}

\author{
Muhammed M. Radhi, ${ }^{a, *}$ and Emad A. J. Al-Mulla ${ }^{b, *}$ \\ ${ }^{a}$ Middle Technical University, Health and Medical Technology College-Baghdad, Iraq \\ ${ }^{b}$ Department of Chemistry, Faculty of Science, University of Kufa, P.O. Box 21, \\ An-Najaf 54001, Iraq
}

Received 04 April 2015; accepted 26 February 2016

\begin{abstract}
A novel self modification of grafted polymer working electrode with carbon nanotubes was success for fabrication from grafting polymer via gamma irradiation and ferrous ammonium sulfate (FAS) as a catalyst. The electrochemical properties of the self modified grafted polymer with CNT (GPESMCNT) improved performance the working electrode at higher conducting surface was done through using in cyclic voltammetry (CV). The GPESMCNT was characterized by surface analytical methods including AFM and SEM. The characterization of electrocnductivity properties of GPESMCNT was studied in $1 \mathrm{M}$ of $\mathrm{KCl}$ with different concentration of $\mathrm{K}_{3}\left[\mathrm{Fe}(\mathrm{CN})_{6}\right]$, at different scan rates, temperature, and different concentrations using $\mathrm{CV}$ technique. The new GPESMCNT improved performance the working electrode in $\mathrm{CV}$ at different techniques such as rotating disc electrode (RDE). also, the nanomaterials in the chain of grafted polymer was enhanced the redox current peaks of $\mathrm{Fe}(\mathrm{II}) / \mathrm{Fe}(\mathrm{III})$ multi times than at commercial working electrodes such as GCE, Pt-electrode, Au-electrode, etc.
\end{abstract}

Keywords: grafted polymer electrode self modified, CNT, cyclic voltammetry, $\mathrm{K}_{3}\left[\mathrm{Fe}(\mathrm{CN})_{6}\right]$.

\section{Introduction}

The modification of grafted polymer with nano-deposits such as $\mathrm{CNT}, \mathrm{C}_{60}$ and activated carbon is very important for the scientists esp. in the electrochemistry by cyclic voltammetric analysis field [1-5].

The unique chemical, physical, electronic (metallic or semiconducting) and high thermal properties of carbon nanotubes (CNTs) made them interesting materials for widespread application in the fields such as electrochemical sensors,

\footnotetext{
*Corresponding author. E-mail address:mmradhi@yahoo.com, imad.almulla@uokufa.edu.iq
} 
biosensors, supports for heterogeneous metal catalysts in organic synthesis, fuel cells, semiconductors, batteries, random access memory cells, field effect transistor, field emission display, atomic force microscopy probes, microelectrodes, specific adsorbents to remove organic pollutants from water and waste water and as a potential drug carriers in cancer therapy[6-9].

Working electrodes must be an electronic conductor and electrochemically inert. Commonly used working solid electrode materials for cyclic voltammetry include platinum, gold and glassy carbon. Other materials (e.g., semi-conductors, for example ITO, indium-tin oxide, or conductive polymers or grafted polymer) are also used, for more specific applications [10,11].

Electrochemical behaviour of famotidine has been studied at composite polymer membrane working electrode. Cyclic voltammetric method has been developed for the determination of drug in pharmaceutical formulation. A well-defined anodic peak was observed for famotidine in the entire $\mathrm{pH}$ range. The current increases steadily with scan rate and concentration. This composite film showed good catalytic behaviour, which includes a good current response. The result is compared with the glassy carbon electrode and it was found that the current with composite polymer electrode is of the order of $18.60 \mathrm{~mA}$ whereas with glassy carbon electrode it was around $565.00 \mu \mathrm{A}[12]$.

Electrochemical study behavior of terthiophene and its corresponding polymer, which is obtained electrochemically as a film by cyclic voltammetry (CV) on platinum electrode. The analysis focuses essentially on the effect of two solvents acetonitrile and dichloromethane on the electrochemical behavior of the obtained polymer. The voltammograms show that the film of polyterthiophene can oxide and reduce in two solutions; in acetonitrile, the oxidation current intensity is more important than in dichloromethane. The impedance plots show the semicircle which is characteristic of charge-transfer resistance at the electrode/polymer interface at high frequency and the diffusion process at low frequency [13].

Grafted copolymer of polypyrrole has been synthesized by electrochemical polymerization of pyrrole in the presence of poly(para-chloromethylstyrene-costyrene-co-pyrrolemethylstyrene). The produced copolymer exhibits an electrical conductivity comparable to that of polypyrrole. This measurement showed that copolymer has excellent thermal stability. The response mechanism of this compound to sense a selection of gases and vapors was investigated, by measuring its electrical conductivity by four-point probe method. This gas sensor may have advantages over the other sensors in its ability to operate at room temperature, lower gas and vapous sensing concentration, suitable solubility, stability in air, sufficient diffusion, and selectivity [14].

This review highlights the recent progress made in the area of thermoelectric (TE) applications of conducting polymers and related composites. Several examples of such materials and their TE properties are discussed. TE properties of new poly(2,7-carbazole) derivatives are highlighted. References are also made to carbon nanotube/polymer composites and their improved electrical and TE performance. Studies on polymer/ inorganic materials composites have also taken a step forward and have shown very promising TE properties [15]. 
In this work, grafted polymer was modified with carbon nanotubes to fabrication grafted polymer electrode self modified with carbon nanotubes. The new grafted polymer electrode was electrochemically characterization in $\mathrm{K}_{3}\left[\mathrm{Fe}(\mathrm{CN})_{6}\right]$ with $\mathrm{KCl}$ aqueous electrolyte by $\mathrm{CV}$ technique

\section{Experimental}

Synthesis of Grafted Polymer modified with carbon nanotubes (GP/CNT)

Polystyrene was grafted with acrylonitrile as a monomer and modified with nano- deposit (carbon nanotubes) and ferrous ammonium sulfate (FAS) as a catalyst using gamma-irradiation. The new grafted polymer modified with carbon nanotubes has been investigated and characterized [3].

\section{Instrument and Electroanalytical Methods}

Electrochemical workstations of NuVant Systems Inc., USA (EZ stat series with potentiostat/glvanostat driven by electroanalytical measuring software) were connected to a PC computer in order to perform cyclic voltammetry (CV), chronoamperometry (CC), and chronoamperometry (CA). An $\mathrm{Ag} / \mathrm{AgCl}$ (3 M $\mathrm{NaCl})$ and platinum wire (1 $\mathrm{mm}$ diameter) were used as the reference and counter electrodes, respectively.

The working electrode used in this study was grafted polymer electrode self modified with carbon nanotubes (GPESMCNT). The voltammetric experiments were carried out with $\mathrm{K}_{3}\left[\mathrm{Fe}(\mathrm{CN})_{6}\right]$ and $\mathrm{KCl}$ as supporting electrolyte. Solution was degassed with nitrogen gas for ten to fifteen minutes prior to recording the voltammogram.

\section{Reagents}

All reagents were analytical reagents or electrochemical grade purity. All solutions were prepared using distilled water. Unless otherwise specified, the supporting electrolyte was used $1 \mathrm{M} \mathrm{KCl}$ in aqueous media at room temperature.

Fabrication the new grafted polymer electrode self modified with CNT (GPESMCNT)

GPESMCNT has been fabricated from grafted polymer modified with carbon nanotubes. The diameter of electrode was $3 \mathrm{~cm}$. A hole was done $(1 \mathrm{~mm})$ to allow $1 \mathrm{~cm}$ length of platinum wire out from other side of electrode. A copper wire was then joined with the platinum wire. After that, all parts of fabricated electrode was covered with glassy tube and then fixed with epoxy resin.

\section{Result and discussion}

Electrochemical properties of grafted polymer electrode self modified with carbon nanotubes (GPESMCNT)

$\mathrm{K}_{3} \mathrm{Fe}(\mathrm{CN})_{6}$ solution is commonly used as a reference standard solution for the purpose of calibrating a voltammetric system in $\mathrm{KCl}$ aqueous solution. During the calibration process of an electroanalytical workstation (EZ stat) using glassy carbon electrode (GCE) and grafted polymer self modified with carbon 
nanotubes electrode (GPESMCNT) as working electrode. The current of $\mathrm{Fe}(\mathrm{II}) / \mathrm{Fe}(\mathrm{III})$ redox couple appears to be significantly enhanced by the GPESMCNT. The enhancement of oxidation-reduction current peaks $+600 \mu \mathrm{A}$ and $-200 \mu \mathrm{A}$, respectively is comparison of GCE at very weak redox current peaks of $+70 \mu \mathrm{A}$ and $-60 \mu \mathrm{A}$ respectively as show in figure $1 \mathrm{a}$ and $\mathrm{b}$.

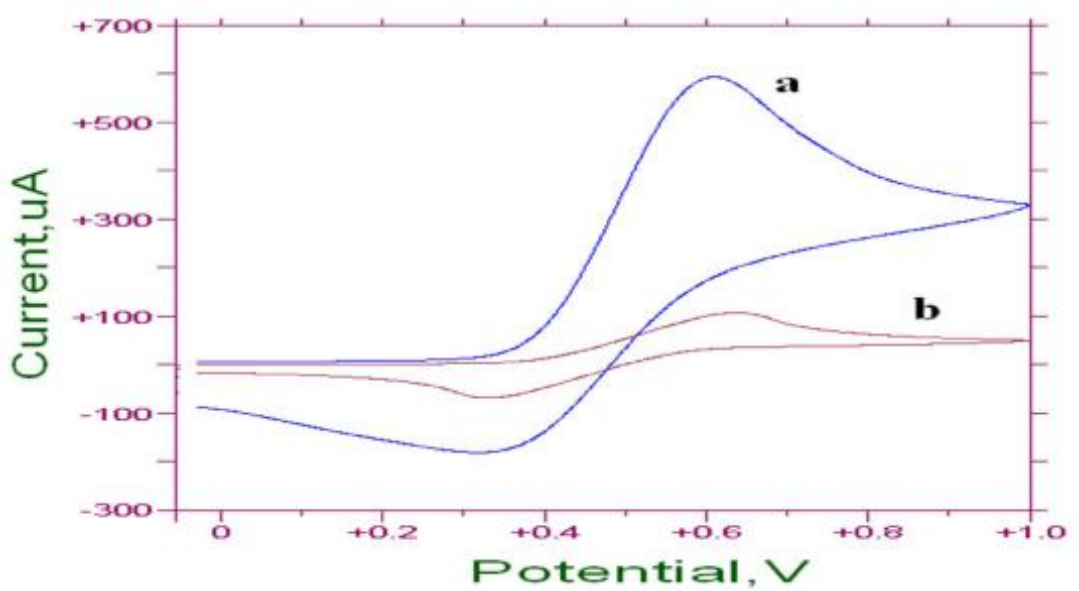

Figure 1. Cyclic voltammogram of $\mathrm{K}_{3}\left[\mathrm{Fe}(\mathrm{CN})_{6}\right]$ in $0.1 \mathrm{M} \mathrm{KCl}(\mathrm{SR}=100 \mathrm{mV} / \mathrm{sec})$ versus $\mathrm{Ag} / \mathrm{AgCl}$ using (a) GPESMCNT and (b) GCE.

\section{Effect of different Scan Rate}

The effect of varying scan rates (SR) on the cyclic voltammograms using grafted polymer electrode self modified with $\mathrm{CNT}$ as working electrode in $1 \mathrm{M} \mathrm{KCl}$ as a supporting electrolyte was studied with $1 \mathrm{mM} \mathrm{K}_{3} \mathrm{Fe}(\mathrm{CN})_{6}$ over a scan rate ranging from $5-1000 \mathrm{mV} / \mathrm{s}$. Oxidation and reduction currents of $\mathrm{Fe}(\mathrm{II}) / \mathrm{Fe}$ (III) couple increased with the scan rate due to heterogeneous kinetics and IR effect. Figure 2 is a reasonably linear dependence of GPESMCNT reduction current on the scan rate and is described by $\mathrm{y}=0.48 \mathrm{x}-1.225, \mathrm{R}^{2}=0.963$. The slope of graph Log Ipc (reduction current) versus $\log (\mathrm{SR})$ is 0.48 ; which is significantly differ from the theoretical value of half for diffusion- controlled process, indicating presence of a complex. The relationship between oxidative potential and scan rate of GPESMCNT, shows a reduction peak at $150 \mathrm{mV}$ in low scan rate but increased to $500 \mathrm{mV}$ at high scan rate (Linearly with $\mathrm{Y}=0.48 \mathrm{X}+150 \quad\left(\mathrm{R}^{2}=0.963\right)$. Surface intercepts process at zero current produces zero current potential $\left(\mathrm{E}_{0,1}\right)$ of 150 $\mathrm{mV}$ for the reduction of GPESMCNT.

\section{Effect of Varying $\mathrm{K}_{3} \mathrm{Fe}(\mathrm{CN})_{6}$ Concentration}

Figure 3 shows the linear current dependent on $\mathrm{K}_{3} \mathrm{Fe}(\mathrm{CN})_{6}$ concentration; observed at concentration range $(5-10 \mathrm{mM})$ which is described by the equation of $\mathrm{y}=18 \mathrm{X}+221.2$ with $\mathrm{R}^{2}=0.984$. The slope of the linear line for $\mathrm{K}_{3} \mathrm{Fe}(\mathrm{CN})_{6}$ showed that a considerably high sensitivity response of $18 \mu \mathrm{A} / \mathrm{mM}$ is readily obtained at GPESMCNT during cyclic voltammetry. 


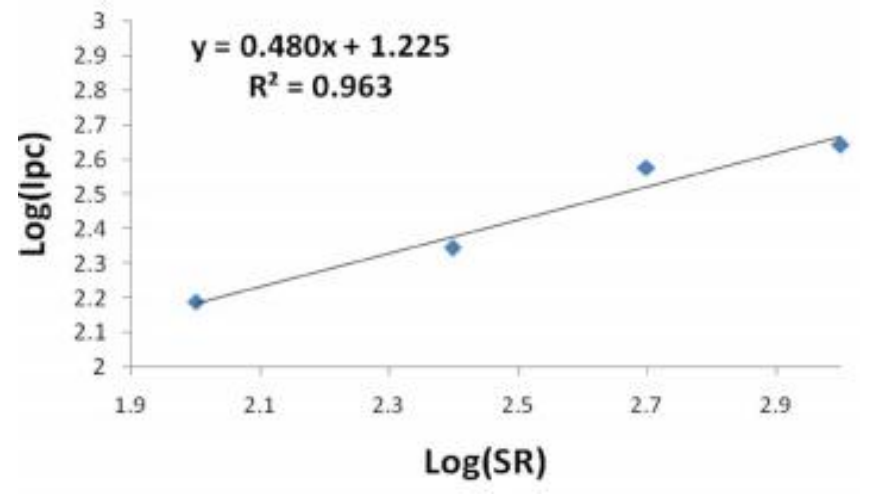

Figure 2. Plot $\log (\mathrm{SR})$ versus $\log (\mathrm{Ipc})$ of $1 \mathrm{mM} \mathrm{K} \mathrm{K}_{3} \mathrm{Fe}(\mathrm{CN})_{6}$ in $1 \mathrm{M} \mathrm{KCl}$ at different scan $\operatorname{rate}(\mathrm{SR}=100,250,500,1000 \mathrm{mV} / \mathrm{sec})$ using GPESMCNT versus $\mathrm{Ag} / \mathrm{AgCl}$ as reference electrode.

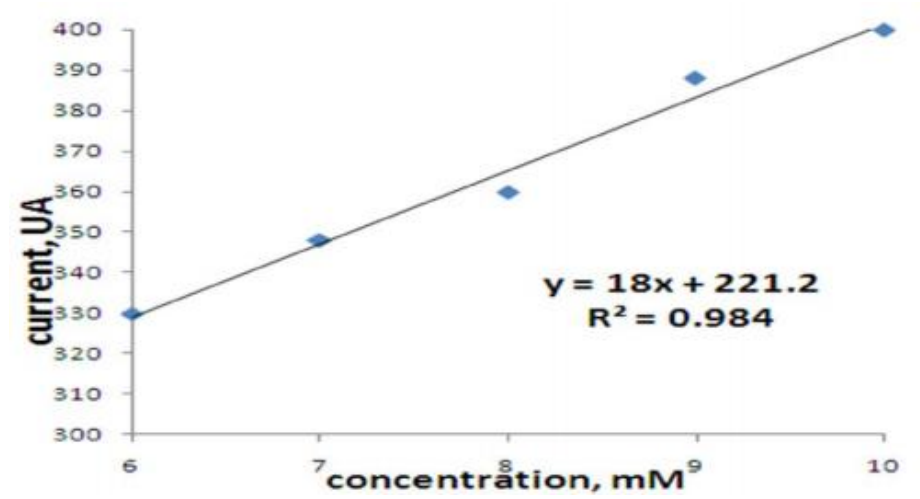

Figure 3. Plot cathodic current versus different concentration of at $\mathrm{K}_{3} \mathrm{Fe}(\mathrm{CN})_{6}$ in $1 \mathrm{M}$ $\mathrm{KCl}$ scan rate $=100 \mathrm{mV} / \mathrm{sec}$ using GPESMCNT versus $\mathrm{Ag} / \mathrm{AgCl}$ as reference electrode.

\section{Reproducibility}

The potential cycling of the redox of GPESMCNT in $1 \mathrm{mM} \mathrm{K}_{3} \mathrm{Fe}(\mathrm{CN})_{6}$ and $1 \mathrm{M}$ $\mathrm{KCl}$ aqueous solution as a supporting electrolyte was carried out during cyclic voltammetry. Continuous potential cycling did not seem to affect the redox current of GPESMCNT as the faradic activity appears reproducible even after 15 cycles, reflecting the stability and reproducibility at the surface of GPESMCNT.

\section{Scanning Electron Microscopy (SEM) of GPE/CNT}

Before electro-analysis, grafted polymer surface appears compact and nonporous. The uniformity of the grafted polymer surface slightly increases since occurrence of protrusion observed phase as shown in Figure4a. After modification with CNT, although many of the nano deposits with homogenous distribution of CNT still remain at about $<1 \mu \mathrm{m}$ as show in Figure $4 \mathrm{~b}$.

\section{Atomic Force Microscopy (AFM)}

The surface image of AFM in an area of $20 \mu \mathrm{m}$ x $20 \mu \mathrm{m}$ of the grafted polymer (polystyrene acrylonitrile) before and after modified with CNT as shown in fig.5. The surface of the electrode appeared to be compact and rough. According to AFM images, the average grain size and thickness of the film were estimated to be $11.23 \mu \mathrm{m}$ and $28.69 \mu \mathrm{m}$, respectively. 


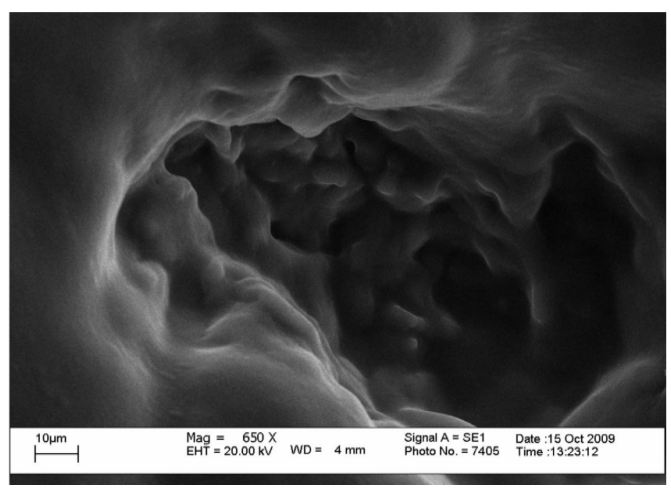

a)

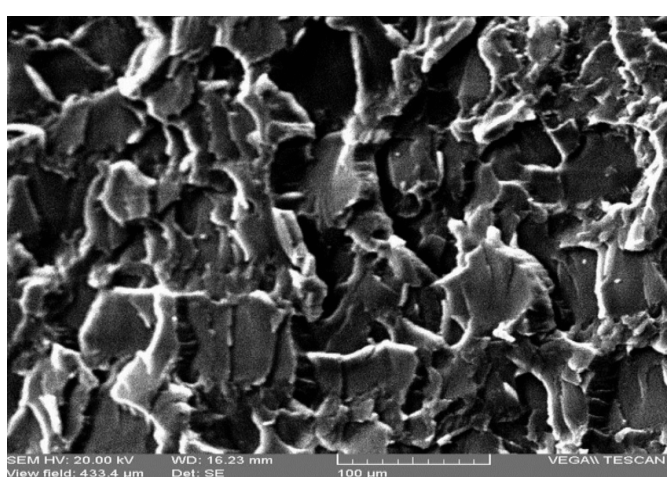

b)

Figure 4. SEM of (a) grafted polymer (b) grafted polymer modified with CNT.
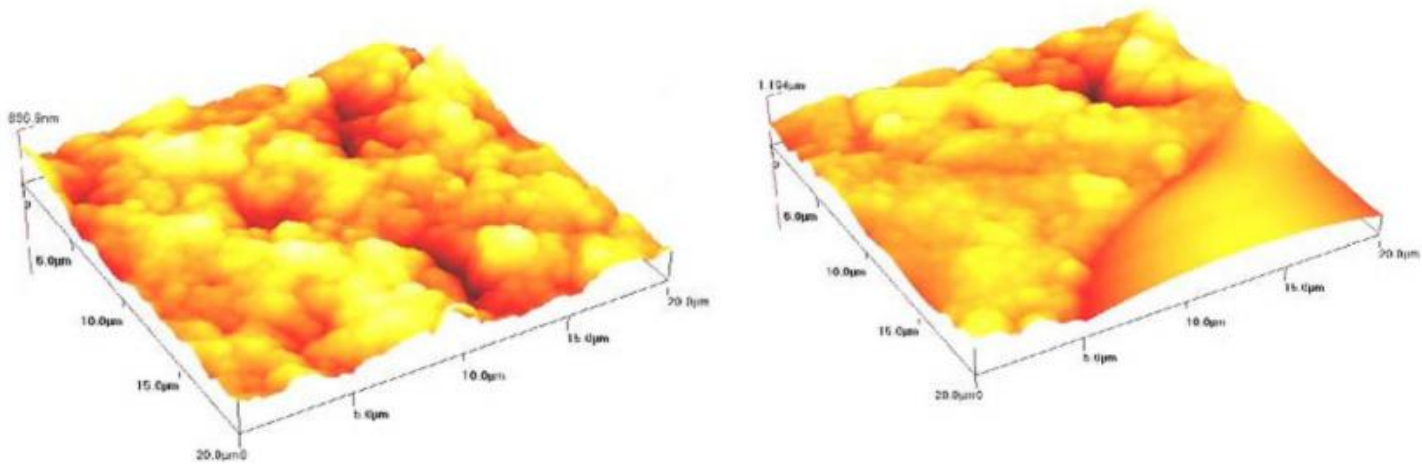

Figure 5. AFM of GPE.

\section{Conclusions}

A Grafted Polymer Electrode self modified with CNT (GPESMCNT) has an extended potential working region as a compared with solid electrodes and classical modification electrodes. The stability of GPESMCNT as a working electrode was evaluated by using $\mathrm{K}_{3} \mathrm{Fe}(\mathrm{CN})_{6}$ in $\mathrm{KCl}$ electrolyte. Redox peaks of $\mathrm{Fe}(\mathrm{II}) / \mathrm{Fe}$ (III) obtained at GPESMCNT showed high current as compared with bar GCE. Electro-catalytic activity of GPESMCNT is therefore evident in this study. GPESMCNT was studied by redox process of $\mathrm{K}_{3} \mathrm{Fe}(\mathrm{CN})_{6}$ in $\mathrm{KCl}$ solution during cyclic voltammetry. The redox peaks potential shifts slightly to less negative value by about $100 \mathrm{mV}$ for oxidative peak and $50 \mathrm{mV}$ for reductive peak with current enhancement of about 3-5 folds. The sensitivity under conditions of cyclic voltammetry is significantly dependent on the concentration and scan rate. Excellent reproducibility of the current is observed, provided a fabricated electrode is used for each experiment without cleaning.

\section{References}

1. Tan WT, Radhi MM, Ab Rahman MZB, et al. J Appl Sci. 2010;10:139-144.

2. Radhi MM, Tan WT, Ab Rahman MZ et al. Sci Research Essays. 2012;7:790-795.

3. Radhi MM, Haider AJ, Jameel ZN, et al. Research J Chem Sci. 2012;2:1-7.

4. Bhattacharya A, Misra BN. Prog Polym Sci. 2004;29: 767-814. 
5. Grande CD, Tria MC, Jiang G, et al. Reactive Funct Polym. 2011;71: 938942.

6. Cha SI, Kim KT, Lee KH, et al. Carbon. 2008;46:482.

7. Lee HJ, Han SW, Kwon YD, et al. Carbon. 2008;46:1850.

8. $\quad$ Lu W, Li N, Chen W, et al. Carbon. 2009;47:3337.

9. Wang L, Zhu D, Duan L, et al. Carbon. 2010;48:3906.

10. Natta G, Corradini P, Bassi I. Nuovo Cimento. 1960;15:68.

11. Ong T, Ng S, Chan H. Polymer. 2003;44:5597.

12. Tiwari DC, Jain R, Sahu G. Indian J Chem Techn. 2008;15:472-475.

13. Maouche N, Nessark B. Int J Electrochem. 2011;Article ID 670513:5 pages.

14. Hoseini SH, Entezami AA. Iranian Polym J. 2005;14:101-110.

15. Dubey N, Leclerc M. J Polym Sci, Part b: Polym Phys. 2011;49:467-475. 